\title{
Optimal Design of Monorail Crane Spring Based on MATLAB
}

\author{
Quanwei Wang, Wenan Kang, Shan Nie \\ Shandong University of Science and Technology,Qingdao,Shandong, 266590,China \\ wqw79279@163.com
}

Keywords: spring; optimal design; MATLAB; mathematical model

\begin{abstract}
This paper studies on optimization design of monorail brake spring, through establishing the mathematical model for optimization design of spring, MATLAB optimization toolbox is used to optimization calculation with examples, to obtain the optimum design parameters. Using optimization design method and MATLAB to improve the design accuracy and efficiency, and provide reliable basis for optimization of other components.
\end{abstract}

\section{Introduction}

Spring is the commonly used elastic parts. Spring design, the traditional method according to the maximum load, maximum deformation and spring structure requirements and other factors, to determine the spring original parameter by experience, after repeated calculation, finally get the design parameters ${ }^{[1]}$. In this paper, taking the minimum volume as spring optimization design goal, establish mathematical model, optimization calculation with the help of MATLAB software, to obtain the optimal parameters of springs, improve the design accuracy.

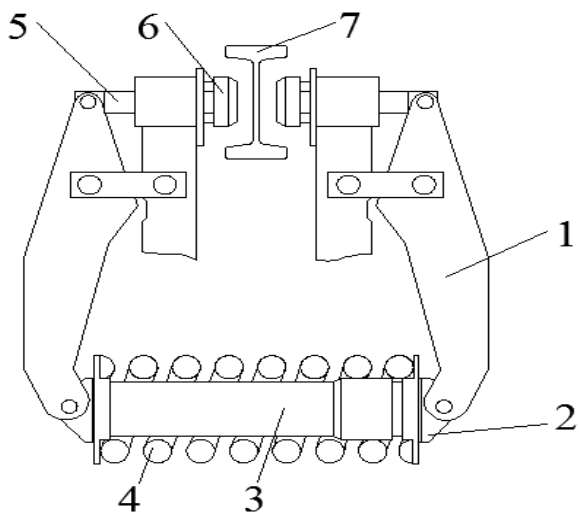

1 , the brake arm 2 , tray 3 , hydraulic cylinder 4 , cylindrical helical compression spring 5 , brake pull rod 6 , friction block assembly 7 , I140E hanging rail

Figure1. The sketch diagram of brake mechanism

The monorail crane locomotive in coal production equipped with a braking device. This article introduces the three poles clamp hydraulic brake structure, as showed in figure 1 . The main process is that spring 4 accumulate energy by its performance, when the brake oil in checking cylinder 3 unloads, brake blocks is pressed onto the track of the web under the spring pressure and then to generate positive pressure, braking is achieved.

\section{Establish the mathematical model}

\subsection{Design Variables}

According to the design requirements,spring material is hot rolled spring steel 50CrMnA.The spring should meet the requirements: service life $N$ ( $N \geq 10^{5}$ cycle), maximum working load $F$ ( $\left.F_{\max }=30 \mathrm{kN}\right)$, stiffness $k(k=84.6 \mathrm{~N} / \mathrm{mm})$, length of the installation $\mathrm{H}\left(H_{0}=900 \mathrm{~mm}\right)$, supporting coils number $n\left(n_{1}=1.5\right)$, and active coils number $n(6 \leq n \leq 17.5)$. Mathematical model of spring as 
shown in Figure $2^{[2]}$. In general, it takes the spring wire diameter $d$, intermediate diameter $D$, active coils numbers $n$ as the design variables. Matrix is used to present spring parameter: $x=\left[x_{1}, x_{2}, x_{3}\right]^{T}=[d, n, D]^{T}$

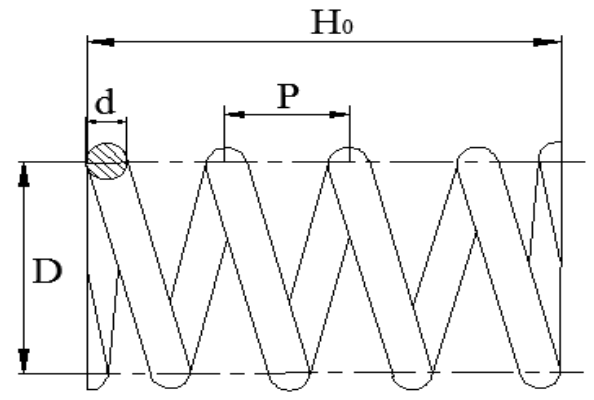

Figure 2. Structure of spring

\subsection{Establishment of Objective Function}

Under the premise of meeting the structure performance, it takes the spring minimum volume as the optimization goal. The objective function is shown as follows:

$$
f(d, n, D)=\frac{1}{4} \pi^{2} d^{2} n D
$$

\subsection{Design constraints}

Constraints generally includes stiffness constrains and strength constraints, vibration stability constraints and spring size constraints.

\subsubsection{Stiffness constraint conditions}

Since the spring stiffness is required to control within a certain range $k_{\min } \leq k \leq k_{\max }, k=\frac{G d^{4}}{8 D^{3} n}$, and $G$ refers to the shear modulus of elasticity of the spring material, thus, stiffness constraint is concluded : $k_{\min } \leq \frac{G d^{4}}{8 D^{3} n} \leq k_{\max }$.

\subsubsection{Strength constraint conditions}

The strength of spring is expressed as:

$$
\tau_{\max }=\frac{8 K D F_{\max }}{\pi d^{3}} \leq[\tau]
$$

In this formula:

$\tau_{\max }$-maximum shear stress, MPa;

$K$-spring curvature coefficient;

$F_{\text {max }}$-maximum working load, $\mathrm{N}$;

The spring curvature coefficient $K$ can be calculated by this formula:

$$
K=\frac{4 C-1}{4 C-4}+\frac{0.615}{C}=\frac{4 D-d}{4 D-4 d}+\frac{0.615 d}{D} \approx 1.66\left(\frac{d}{D}\right)^{0.16}
$$

Where $C$ refers to spring convolute ratio, $C=\frac{D}{d}$, take formula (2) into formula(3) to figure out strength constraint condition:

$$
\frac{4.23 F_{\max } D^{0.84}}{d^{2.84}}-[\tau] \leq 0
$$

\subsubsection{Stability constraint conditions}

For the compression spring, in order to avoid the occurrence of instability, the ratio of its height to diameter $b$ should not exceed the allowable value $[b]$, namely: $b=\frac{H_{0}}{D}=\frac{n p+1.5 d}{D} \leq[b]$. Here, take the spacing length of spring $p=0.35 D$ and $[b]=5.3$, so stability constraint conditions is deduced : $0.35 D n+1.5 d-D[b] \leq 0$. 


\subsubsection{Convolute ratio constraint conditions}

Convolute ratio $C, C=\frac{D}{d}, 4 \leq C \leq 8,\left\{\begin{array}{l}D-8 d \leq 0 \\ 4 d-D \leq 0\end{array}\right.$

\subsubsection{Spring maximum deformation constraint}

The maximum spring deformation length $\lambda_{\max }$ can not exceed the total axial spacing. It can be expressed as:

$$
\begin{aligned}
& \lambda_{\text {max }} \leq n \delta \quad \delta=p-d \\
& \lambda_{\text {max }}=\frac{F_{\max }}{k}=\frac{8 D^{3} n F_{\text {max }}}{G d^{4}}
\end{aligned}
$$

In the formula, $\delta$ refers to axial spacing, bring $p=0.35 D$ into above formula to conclude deformation constraint condition:

$$
\frac{8 D^{3} n F_{\max }}{G d^{4}}+n(d-0.35 D) \leq 0
$$

In summary, the mathematical model could be established as follow:

Spring parameter matrix: $x=\left[x_{1}, x_{2}, x_{3}\right]^{T}=[d, n, D]^{T} \quad$ objective function: $f(x)=d^{2} n D=x_{1}^{2} x_{2} x_{3}$ constraint condition: $g_{1}(x)=84.6 x_{3}^{3} x_{2}-9875 x_{1}^{4} \leq 0$

$$
\begin{aligned}
& g_{2}(x)=1.269 \times 10^{5} \frac{x_{2}^{0.84}}{x_{1}^{2.84}}-570 \leq 0 \\
& g_{3}(x)=0.35 x_{2} x_{3}+1.5 x_{1}-5.3 x_{2} \leq 0 \\
& g_{4}(x)=3.05 \frac{x_{2}^{3} x_{3}}{x_{1}^{4}}+x_{3}\left(x_{1}-0.35 x_{2}\right) \leq 0 \\
& g_{5}(x)=x_{2}-8 x_{1} \leq 0 \\
& g_{6}(x)=4 x_{1}-x_{2} \leq 0
\end{aligned}
$$

\section{Optimization Design based on MATLAB Optimized Tool Box}

\subsection{Calculation of Function Optimization}

Fmincon function attempts to work out the minimum of a constrained nonlinear multi-variable function. The process of optimization calculation by fmincon function are as follows ${ }^{[3]}$ :

$\min f(X)$

$$
\begin{aligned}
& c_{i}(X) \leq 0 \\
& \text { ceq }_{i}(X)=0
\end{aligned}
$$

S.t. $\quad A(X) \leq b$

$$
\begin{aligned}
& \operatorname{Aeq}_{i}(X)=b e q \\
& l b \leq X \leq u b
\end{aligned}
$$

Where $X, b, b e q, l b$, and $u b$ are vectors, $A$ and Aeq are matrices, $c(x)$ and $c e q(x)$ are functions, and $f(x), c(x)$, and $c e q(x)$ could be nonlinear function ${ }^{[4]}$.

The optimization calculation format is:

[x,fval,exitflag]=fmincon(fun, $\mathrm{x}_{0}, \mathrm{~A}, \mathrm{~b}$, Aeq, beq,lb,ub,nonlcon,options)

Where the fval refers to the minimum value of the objective function, the exitflag refers to iteration termination condition, and fun refers to the objective function. $\mathrm{x}_{0}$ is taken as the primary value to search the minimum. Besides, A refers to a matrix on linear formula coefficients , $b$ refers to a matrix on the result of linear formula,and lb refers to the matrix of the bottom boundary of parameters. Option refers to specified optimization parameters option, nonclon refers to the function about constraints and options applied to large-scale algorithm or medium-scale algorithm ${ }^{[5]}$. 


\subsection{Optimization Calculation Process}

(1) Establish an M-file for objective function:

The expression on objective function: $\quad f=x(1) \wedge 2 * x(2) * x(3)$

(2) Establish an M-file for constraint function:

Establish an M-file by nonlcon: function[c,ceq]=mycon(x)

$\mathrm{c}(1)=84.6 * \mathrm{x}(2) * \mathrm{x}(3)^{\wedge} 3-9875 * \mathrm{x}(1)^{\wedge} 4$;

$c(2)=126900 * x(2) \wedge 0.84-590 * x(1)^{\wedge} 2.84$;

$\mathrm{c}(3)=3.05 * \mathrm{x}(2)^{\wedge} 3 * \mathrm{x}(3)-\mathrm{x}(1)^{\wedge} 4 * \mathrm{x}(3) *(0.35 * \mathrm{x}(2)-\mathrm{x}(1))$;

ceq $=0.35 * x(2) * x(3)+1.5 * x(1)-900$;

(3) Calculation program:

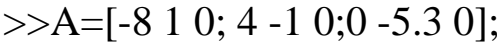

$>>b=\left[\begin{array}{lll}0 & 0 & -900\end{array}\right]$

$>>\mathrm{x}_{0}=\left[\begin{array}{lll}30 & 180 & 16\end{array}\right]$;

$>>\mathrm{lb}=$ zeros $(3,1)$;

>>Options=optimset('largescale', 'off');

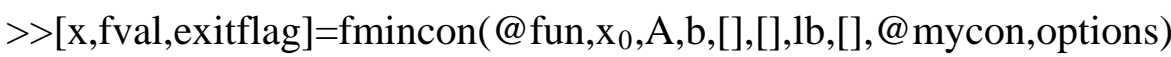

(4) Operational results:

According to the function: [x,fval,exitflag] $=$ fmincon(fun, $\mathrm{x}_{0}, \mathrm{~A}, \mathrm{~b}, \mathrm{Aeq}, \mathrm{beq}, \mathrm{lb}, \mathrm{ub}$, nonlcon,options) $\mathrm{x}=[30.2674198704009169 .811320754717015 .2202349588180]$

fval $=2.367766105318849 \mathrm{e}+006$

exitflag $=4$

\subsection{Results analysis}

Comparison between conventional design and MATLAB optimization results is as follows:

Table.1 Comparison of two design methods results

\begin{tabular}{|c|c|c|c|c|}
\hline \multirow{2}{*}{ design method } & \multicolumn{3}{|c|}{ design parameter } & $\begin{array}{c}\text { objective } \\
\text { function }\left(\mathrm{mm}^{3}\right)\end{array}$ \\
\cline { 2 - 4 } & $D(\mathrm{~mm})$ & $d(\mathrm{~mm})$ & $n$ & 2592000 \\
\hline Traditional design & 180 & 30 & 16 & 2368114.82 \\
\hline Optimal design & 169.81 & 30.27 & 15.22 & \\
\hline
\end{tabular}

The

result

exitflag is bigger than zero, which means that the solution is convergent and efficient ${ }^{[6]}$. As can be

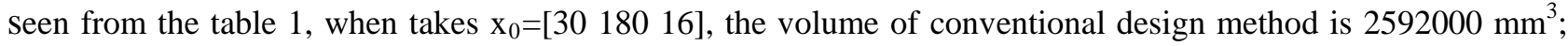
and the optimized volume is $2368114.82 \mathrm{~mm}^{3}$, it is $91 \%$ of the volume by traditional way. The final design data can be adjusted to the standard series parameters in the light of the requirements.

\section{Summary}

To sum up, in the spring design, spring design method based on MATLAB optimization toolbox can effectively improve the design accuracy, and design the spring of optimal combination parameters , the method is the improvement of traditional design.

\section{Acknowledgments}

This study has been supported by innovative education project for graduate of Shandong province, Shandong University of Science and Technology science and technology fund for graduate creative research (grant No. YC140319). The authors would like to express their sincere 
thanks to these fund sponsors.

\section{References}

[1]. Cheng Daxian, Machine design handbook, Spring (Fifth Edition )[M], Beijing: China Chemistry Press, 2010.

[2]. Ye Yuanlie, Mechine Design Theory and Design [M] Beijing: Tsinghua University Press, 1994.

[3]. Yu Runwei \& Zhu Xiaohui, MATLAB Fundamentals and Applications [M], Mechanical Industry Press, 2008: 85-91.

[4]. Li Wanxiang, Engineering optimization design and MATLAB realization [M] Beijing: Tsinghua University Press, 2010.

[5]. Gong Chun \& Wang Zhenglin, Proficient MATLAB optimal calculation [M]. Electronic Industry Press, 2009.

[6]. Wang Guoqi, Mechanical Optimization Design [M] Beijing: Mechanical Industry Press, 2009. 